\title{
VISION
}

\section{Fisheye views}

Archerfish eyes must cope with the light spectrum of two very different media. These fish live among mangroves and in rivers, and are renowned for their ability to bring down an insect target from overhanging foliage by spitting a stream of water at their prey, as pictured here. Shelby Temple and colleagues have investigated the visual pigments and spatial resolving power of archerfish eyes, and present the results in the context of the requirements for vision at the water-air interface (S. Temple et al. Proc. R. Soc. Lond. B doi:10.1098/ rspb.2010.0345; 2010).

Using microspectrophotometry, the authors find that - as is known in other species - the pigments and spectral tuning vary between different parts of the retina (in this case, subdivided into dorsal, ventro-nasal and ventro-temporal regions). They interpret these variations in terms of the tasks the eye has to perform when operating along three visual axes; that is,

three directions in which the eye might look.

A simplified description of their conclusions is that the dorsal retina has maximum spectral sensitivity at 454 and 570 nanometres, a combination that Temple et al. consider is well suited for discriminating between shades of brown, and for identifying objects

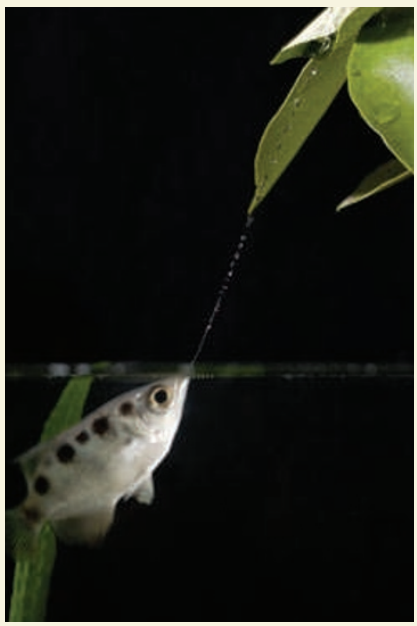

beneath them. The values for the ventro-nasal retina (visual axis up and behind) are $502 \mathrm{~nm}$ and $620 \mathrm{~nm}$. The 502-nm peak is tuned, the authors suggest, for detecting dark images against a background of bright sky, such as the silhouette of an aerial predator.

The ventro-temporal retina presents peak sensitivities at $453 \mathrm{~nm}, 535 \mathrm{~nm}$ and $565 \mathrm{~nm}$. This combination is possibly used for colour vision along the visual axis appropriate for sighting prey against a background of foliage. From video recordings, Temple et al. conclude that this part of the retina aligns with spitting angles, and they estimate that the visual resolution allows an archerfish to tell the difference between two objects $2 \mathrm{~mm}$ apart at a range of $550 \mathrm{~mm}$.

Archerfish can be trained to spit at coloured targets. That, say Temple et al., makes them excellent subjects for investigating further aspects of the function of intra-retinal differences. Tim Lincoln
20 of the mutations was comparable for all three tumours, that 26 showed increased prevalence in the xenograft and/or the metastatic tissue, and that the prevalence of 2 was significantly decreased relative to the primary tumour. This suggests that at least three cell clones from the primary tumour carried over into the metastatic and xenograft tumours: one carried mutations that decreased in prevalence, one had mutations that increased in prevalence, and one carried mutations whose prevalence did not change compared with the primary tumour. This metastasis therefore does not seem to have formed from a single cell, but rather from a cell population that, in this case, contained at least these three clones.

Another remarkable result was that 16 of the 20 mutations present at increased prevalence in the metastatic tumour were also present with higher prevalence in the xenograft. This pattern of concordant selection of one or more clones carrying common mutations during progression to metastasis and establishment of a xenograft suggests that similar evolutionary pressures are at work on these cells in both environments. This may provide some indication of the aspects of the metastatic process that are influenced by the aberrations carried in the selected clone, because some processes needed for metastasis, such as invasion and barrier penetration, may not be important selective forces in the xenograft environment. These aspects of metastasis therefore may not be influenced by aberrations that are selected in both xenograft and metastasis.

Of course, Ding et al. ${ }^{4}$ assessed the evolution of only one tumour. But if their results can be reproduced in larger studies, comparative investigations of primary-tumour/xenograft/ metastasis triplets might facilitate identification of genomic aberrations that play a significant part in the pathophysiology of the tumour and metastasis, and provide clues about the biological roles of these genomic regions. They could also provide information about clonal diversity in metastatic lesions, which may help to identify subpopulations of cellular molecules, and thus influence cancer therapy.

Ding and colleagues' data therefore hint that future sequencing of some metastatic-cancer genomes should be considerably deeper than contemplated at present to allow statistically robust estimates of mutation prevalences to be obtained. Moreover, functional assessments of the affected genomic regions will be needed to determine which genes in the selected clones are drivers and which mere passengers. Concordant selection of mutations in the metastatic and xenograft tumours could provide initial clues to the most useful candidates for functional assessment.

Joe Gray is in the Lawrence Berkeley National Laboratory, Life Sciences Division, Berkeley,

California 94720, USA.

e-mail:jwgray@lbl.gov

Mardis, E. R. \& Wilson, R. K. Hum. Mol. Genet. 18 R163-R168 (2009).

2. Metzker, M. L. Nature Rev. Genet. 11, 31-46 (2010).

3. Morozova, O., Hirst, M. \& Marra, M. A. Annu. Rev. Genomics Hum. Genet. 10, 135-151 (2009).

4. Ding, L. et al. Nature 464, 999-1005 (2010).

5. Nguyen, D. X., Bos, P. D. \& Massagué, J. Nature Rev. Cancer 9, 274-284 (2009).

6. Gupta, G. P. \& Massagué, J. Cell 127, 679-695 (2006)

7. DeNardo, D. G., Johansson, M. \& Coussens, L. M. Cancer Metastasis Rev. 27, 11-18 (2008)

8. Langley, R. R. \& Fidler, I. J. Endocr. Rev. 28, 297-321 (2007)

See also News Feature, page 972.

\section{BEHAVIOURAL ECOLOGY}

\section{Ways to raise tadpoles}

\section{Hanna Kokko and Michael Jennions}

\section{To reduce parental care, just add water - that's the conclusion of an intriguing investigation into the extent of the motherly and fatherly devotion that different species of frog extend to their offspring.}

Nature documentaries frequently invite their viewers to contemplate that only a tiny minority of the perfectly formed larvae floating in the sea, or crawling on land, can ever hope to make it to the adult stage. Parents of many species seem surprisingly unconcerned, leaving their progeny to fend for themselves. In some species, however, parents provide their offspring with costly, time-consuming services, ranging from protecting them against predators and environmental stresses to giving them shelter or food.

Why does this diversity of solutions exist? Theoreticians state that parents may reduce care to increase the number of young produced and/or to improve the parents' own survival. Both trade-offs reduce the lifetime fecundity of parents, so parents are likely to provide care 